\title{
An Internet of Things Approach for RFID Smart Tollgate Automation
}

\author{
Poorani. P, Kiruthika. K, Lavanya. A \\ Assistant Professor, Department of Electrical Communication Engineering, \\ Karapaga Vinayaga College of Engineering and Technology, Tamil Nadu, India
}

\begin{abstract}
In today's era vehicles area unit giant in variety, the cubicle appears to become bottleneck to meet up with the gates because of their manual operations. This process may take few minutes to pass the toll system. In order to decrease this passing time, the process of toll system can be automated by technology enablement of Internet of Things (IoT) and identification of vehicle with the help of radio frequency. Automated Toll Collection System used for collecting tax automatically. A vehicle will hold an RFID tag. This tag is nothing however distinctive positive identification allotted. This will be assigned by RTO or traffic governing authority. As vehicles don't have to stop in a queue, it assures time saving and fuel conservation. This approach would reduce the manual work and hence would make the passing of the vehicles much faster as compared to traditional toll system and also introduce identification system for vehicles against which stolen and accident cases.
\end{abstract}

Keywords: Toll Booth, RFID, Arduino, IoT, Cloud Computing, WiFi Module, Firebase database

\section{INTRODUCTION}

Automation has made its way into our lives and is slowly seeping to our lifestyle. We see wide spectra of places where this can be used for the betterment of the society. The number of vehicles on the road is increasing at an alarming rate because of which the condition of the roads is worsening quickly. Then major roads have manual toll systems, where every vehicle has to stop and pay for toll tax and then leave the lane. With enormous increase in number of vehicles on road, the process of traditional toll system has become worst and at times on popular routes the waiting times of vehicles is significantly high. So the objective was to give a solution of this delayed manual toll system by designing and implementing an automatic tolling framework for gathering toll. The system which is better in terms of accuracy, efficiency and cost effectiveness with lesser delay for vehicles. So such systems are the need of society now a day's to speed up the toll passage process.

In our day to day life, we tend to pay certain quantity of tax through tract to the govt. The toll gates area unit principally found on national highways and bridges etc., and that we pay standing over a queue within the kind of money though the quality of vehicles gets interrupted by this methodology that takes longer period of time, additional consumption of fuel and conjointly pollution level get magnified in this region, rather than that the tactic ordinarily employed by industries and in advanced countries is that the Electronic Toll assortment System. Electronic toll assortment system is that the technology that allows the automated electronic toll assortment from the pocketbook account registered on the name of car owner, crucial whether or not the vehicle is registered or not and informs the toll authorities avoiding toll violations. Over last decades, electronic toll collection system has been implemented in United States and many other countries with a new improvement in it.

The automatic toll e-ticketing system is the approach used for the vehicle when it reaches the toll plaza, this is detected by using RF ID is used to read each vehicle with the help of
RF ID receiver. This then transmits the vehicle number through the RF ID transmitter located in vehicle. We assume that vehicles have identification numbers. The read the signal and information about vehicles owners. These RF signals area unit received by associate $\mathrm{RF}$ receiver at the parcel that send knowledge to a computer's port. A computer code program running on the pc retrieves vehicle details from its vehicle information.

Depending on this information, applicable toll tax is subtracted from the pre-paid account of the vehicle's house owners.The owner receives AN SMS message on his/her mobile regarding the main points of the payment. If the balance in the owner's account is low or if the 3 vehicle is not equipped with an RF system, the toll gate remains close. This is very simple method for enhancing the performance of infrared electronic-toll collection systems, in such a case, the vehicle owner will have to recharge the toll collect the receipt.

Thus providing good quality transportation will significantly contribute to better economy and productivity. Cost reduction in transporting raw material to factories and moving finished products to consumer market is an important factor in economic and social growth. To reduce congestion and travel time, increase safety, increase efficiency are some important challenges that are faced. Hence by increasing system's efficiency and making an efficient utilization of modern technology, one can achieve better optimization of the current constraint resources. IoT Toll Collection systems are aimed at automating the toll plazas so as to maintain fairness in the transactions during toll collections. This literature paper presents the idea of IoT Toll Collection systems.

IOT (internet of things) based mostly toll booth manager system is such sort of a system that has the automated toll automated toll assortment method with assistance of web resources and RFID (radio frequency identification) technology. If we tend to state responsibility, then 
manually toll assortment method is incredibly difficult as a result of it's not most simple to four manage hundred and thousands of vehicles at same time. It needed additional man power further like additional toll assortment booth that can be most expensive and tough for toll assortment department. On alternative hand, most time is wasted by the vehicle homeowners after they square measure substitute most long toll assortment row.

\section{EXISTING WORK}

Aniruddha Kumawat ET. Al. an Automated Toll Collection System using RFID used for collecting tax automatically. Radio frequency is used for identification. A vehicle will hold an RFID tag. All the basic information of the vehicle will be stored in this, along with the amount paid by the vehicle at the toll booth. Whenever a vehicle will pass the toll booth, the readers which will be placed will read the vehicle and also deduct the necessary amount of cash. The new balance would be updated after the transaction gets over. If the balance is insufficient, the net total amount I the card goes to negative. This may lead to some financial loss to the system. So negative balance can be an escape for anunauthentic vehicle.

According to Khadijah Kamarulazizi and Widad Ismail, this paper focuses on an electronic toll collection (ETC) system using radio frequency identification (RFID) technology [2][4]. This method automated the system and eradicated the need and hassle of manually paying the toll. Data information are also easily exchanged between the vehicle and the authority that is collecting the tax, thereby enabling a more efficient toll collection by reducing traffic and eliminating possible human errors. But this was limited to the local servers, so scalability and reach upto all tolls was not possible.

According to Etqad Khan, Dipesh Garg, Rajeev Tiwari and Shuchi Upadhyay, this paper focus on to reduce d reduce the manual work and hence would make the passing of the vehicles much faster as compared to traditional toll system.Every vehicle is tagged with a RFID tag, which has vehicle's registration number in it, which can be sensed by RFID reader present at tollbooth.RFID reader will send this information to IoT controller (Arduino). Sensed registration number can looked in to cloud database for getting wallet balance and if sufficient balance is there, and then toll charges can be deducted automatically. While rider can enjoy pause free ride and will be intimated about his trip deduction charges. For interaction of client a mobile app will also be designed with which client can track all logs of payments and can add money in wallet using the app. The sole purpose is to reduce the hardships caused by manual toll collection system and it assures time saving, fuel conservation and contributing in saving of money by making process automatic.

\section{DESIGN OF SYSTEM}

\section{A. Radio Frequency Identification (RFID)}

RFID is used for identification and uses electromagnetic field in the process. RFID system consists of two components, RFID tag and RFID reader.The RFID tags that are available in the market electronically store information. RFID tags are of two types- a) Active RFID tags b) Passive RFID tags. Passive RFID tags do not have a battery of their own and extract all its powers from the radio waves of the RFID reader. Here is where an active RFID tag differs from a Passive one because an active RFID tag has its own battery and can be detected by a reader from hundreds of metres away. RFID is one method for Automatic Identification and Data Capture. RFID reader is used to reading the tags, collecting the stored information and then communicating the result to a IoT controller or to database. Reader communicates with tag using its own antenna. This Radio Frequency Identification has found widespread use in the recent times and has been the centre of development when talking about the Internet of Things. RFID tag used for this work is as shown below in Figure no 1.

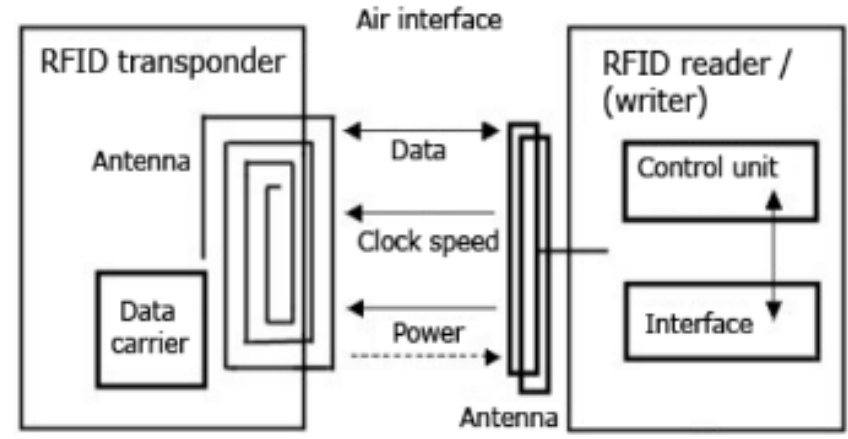

Fig 1: RFID Tag Used

\section{B. Arduino Microprocessor}

Arduino is an Open Source enterprise that aims at making this world a better place by producing single board microcontrollers and microcontroller kits that can be programmed to develop devices that are endowed with abilities to interact with objects from the non-digital world. Various microprocessors and controllers are part of different Arduino designs. The board provided has numerous Input/output pins that can be used to interface with different devices and boards. It consist of programmable circuit board and integrated development environment which is used to write the code and then feed the code to circuit board. Arduino can be coded for a designed function or a specific task. The Arduino has USB port that lets us connect it with the computer and in turn is used to upload programs on it. The language used for coding is generally $\mathrm{C}$ and $\mathrm{C}++$ and all the embedded libraries are also available. Arduino also provides a dedicated integrated development environment (IDE) based on the Processing language project for compilation and upload of our Sketch (Program) on the board. It has 6 analogue input pins and 14 digital I/O pins.

\section{Wi-Fi Module ESP 8266}

$\mathrm{Wi}-\mathrm{Fi}$ is the phenomenon in which computer or other devices communicate with one another. The ESP8266 is a Microcontroller unit capability. It is a low cost Wi-Fi and it comes with the TCP/IP stack fully embedded.It has a micro USB port for power, programming and debugging operations. This module is connected with Arduino kit as shown in Figure 2.

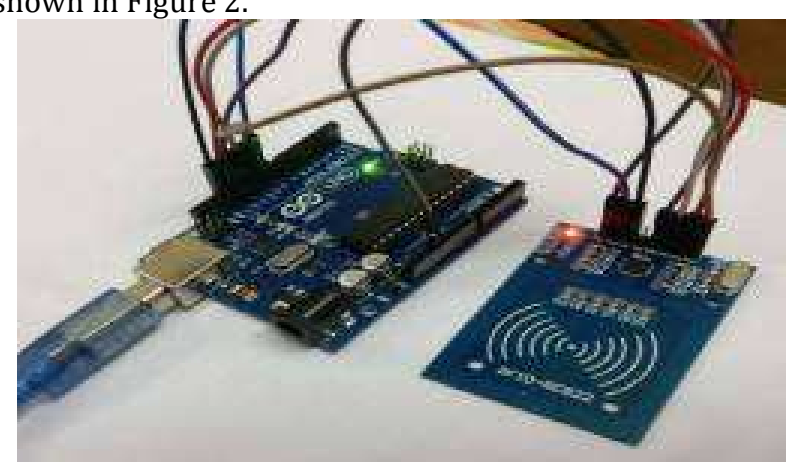

Fig 2: Arduino connected with WiFi Module for sending data to cloud database server 


\section{Mobile App}

A mobile app is developed in this system, which can connect every user with toll database. User can see the tolls on his way and can check the balance in his wallet. As his vehicle passes from toll gate, automatic his vehicle registration number is read and payment from his wallet balance is deducted. Receipt of toll charges is generated and can be downloaded from his mobile application user login controls. Then using app user can add balance into wallet.

\section{E. Use of Sensors}

Use of Radio Frequency Identification as a means to see which vehicles are registered and which are not. RFID tags are installed on each vehicle and when the vehicles close in on the gate, the RFID reader strategically placed, does read the tag and checks on the basis of the initiation made. RFID uses Electromagnetic field in the process of Identification. The cards used in this process can either be Read-only or Read write depending upon the cost structure. The Readonly RFID tags cannot hold any data but the Read-write tags can hold up to $128 \mathrm{~B}$ of data. The RFID card have a unique Identification number which are used for checking whether a given vehicle is registered or not. This section holds major significance because it decides that whether the vehicle entering is valid or not and then proceeds for the pay.

\section{F. Data Acquisitions}

Every RFID tag has a unique Identification number and it is unique for all the cards. As we will recognise the vehicle by the tag/card embedded in the vehicle so at the time of vehicle's registration, it is must that every vehicle must be associated with a RFID tag.In this way,every vehicle will have a unique identity that can be easily identified by the RFID reader. The RFID reader in turn, reads all the RFID tags to fetch their unique ID numbers, and the Arduino is programmed to only accept certain RFID tags, thus allowing only the vehicles with the registered tags on them.The moment the tag comes within magnetic field of RFID reader,current is induced in it and it get energised.

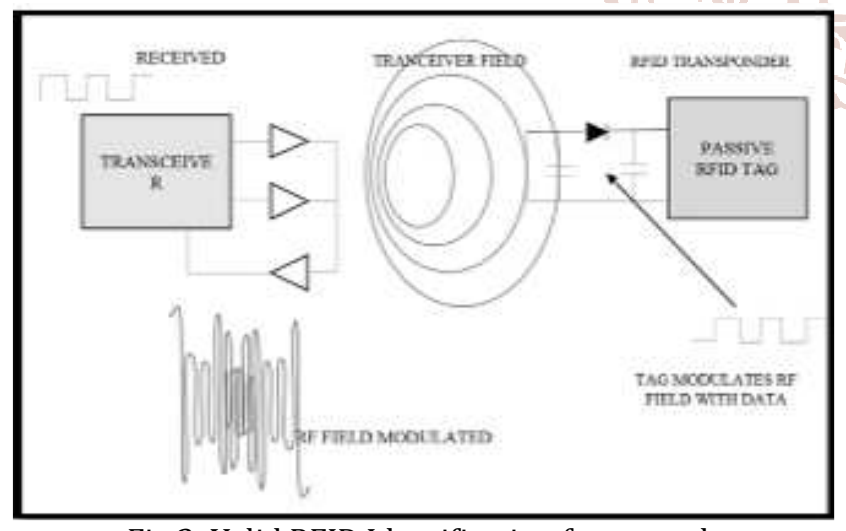

Fig 3: Valid RFID Identification framework

Thus stored information in tag is sent to reader. Then the designed system authenticates the user and allows the person to pass through the toll after deducting a fixed amount. All information of user is stored in cloud. The database containing all the details of the vehicles is set up on Firebase cloud service. With every unique Identification number, is associated three other fields, namely, Name of the owner, Registration number of the car and the account balance for the toll payment. From here on, this data is fetched and further analysis on the data is performed.

\section{G. Internet of things}

The ESP8266 may be a cheap Wi-Fi micro chip with full TCP/IP stack and microcontroller capability created by Shanghai-based Chinese manufacturer, Expressive Systems The chip initial came to the eye of western manufacturers in August 2014 with the ESP-01 module, created by a third-party manufacturer, Ai-Thinker. This tiny module permits microcontrollers to attach to a Wi-Fi network and build straightforward TCP/IP connections mistreatment Hayes-style commands. However, at the time there was nearly no English-language documentation on the chip and therefore the commands it accepted. The terribly low worth and therefore the proven fact that there have been only a few external elements on the module that advised that it might eventually be terribly cheap in volume, attracted several hackers to explore the module,chip and therefore the software package on that likewise on translate the Chinese documentation. The ESP8285 is associate ESP8266 with one $\mathrm{MiB}$ of intrinsical flash, leaving single-chip devices capable of connecting to $\mathrm{Wi}-\mathrm{Fi}$.
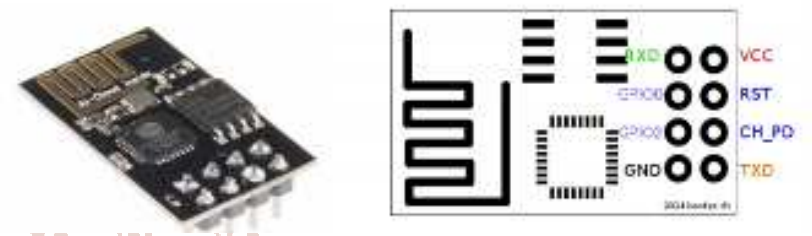

Fig 4: ESP8266 and pin diagram

\section{PROPOSED WORK}

With the many development in Roadways, there's a rise within the range of toll plazas. These toll plazas have long queues and therefore the time consumed in paying money and returning modification causes all the additional delay. The system designed for an IOT primarily based Toll booth Manager System during which an individual will use ANRFID to pay the Toll charge. Once the RFID is swiped, the system would check if it's sufficient balance and so deduct the toll charge and update the balance through IOT. Internet of Things (IoT) is largely the network of 'things' by that physical things will exchange knowledge with the assistance of sensors, physical science, package and property. These systems don't need any human interaction. IoT Technology is found in several industries like health care, Energy, Transportation etc. during this project, the info of the RFID card is distributed to the web site through IOT.

IOT primarily based Toll Booth manager system helps the toll booths to gather the toll by simply swiping a card. The project consists of 4 keys by that the user will choose the mode of toll assortment. Once the RFID mode is chosen, the alphanumeric display displays the message to indicate the RFID card. the cardboard must be then swiped on the cardboard Reader. When the cardboard is swiped, the micro-controller: Arduino can check if the cardboard is valid or not. If the cardboard is valid then the microcontroller can check if the cardboard has sufficient balance. The cardboard balance is showed on the alphanumeric display display. Once the micro-controller detects sufficient balance, the toll gate is opened and therefore the vehicle is allowed to undergo.

With the significant development in Roadways, there is an increase in the number of toll plazas. These toll plazas have long queues and the time consumed in paying cash 
and returning change causes all the more delay. We have designed an IOT based Toll booth Manager System in which a person can use an RFID to pay the Toll charge. When the RFID is swiped, the system would check if it has sufficient balance and then deduct the toll charge and update the balance through IOT. Internet of Things (IoT) is basically the network of 'things' by which physical things can exchange data with the help of sensors, electronics, software and connectivity. These systems do not require any human interaction. IoT Technology is found in many industries like healthcare, Energy, Transportation etc. In this project, the data of the RFID card is sent to the website through IOT.

IOT Based Toll Booth manager system helps the toll booths to collect the toll by just swiping a card. The project consists of four keys by which the user can select the mode of toll collection. Once the RFID mode is selected, the LCD displays the message to show the RFID card. The card has to be then swiped on the Card Reader. After the card is swiped, the micro-controller: Arduino will check if the card is valid or not. If the card is valid then the micro-controller will check if the card has sufficient balance. The card balance is displayed on the LCD display. Once the micro-controller detects sufficient balance, the toll gate is opened and the vehicle is allowed to pass through. This method uses an IR-sensor i.e. Infrared Sensor to indicate that the vehicle has crossed the toll gate and the gate will be closed. The advantages of the system is 1 . IOT based mostly Toll Booth Manager System may be used the least bit the parcels on the Highways victimization this project would facilitate the vehicle to pass the toll plaza no end that successively would facilitate folks save plenty of their time.2. This project can even be used at duty assortment booths.

A frequency identification reader may be a device accustomed gather info from an RFID tag, that is employed to trace individual objects. Radiowaves. RFID may be a technology similar in theory to bar codes. AN infrared receiver is hardware that sends info from AN infrared remote to a different device by receiving and secret writing signals. In general, the receiver outputs a code to unambiguously establish the infrared signal that it receives. This info area unit controlled by arduino controller, then it'll send to the motor (for gate) via motor driver, that is employed to spice up up the input voltage. LCD shows the gathering info, if there's any drawback the buzzer can sound up aggregation info can update to the website by victimization IOT.

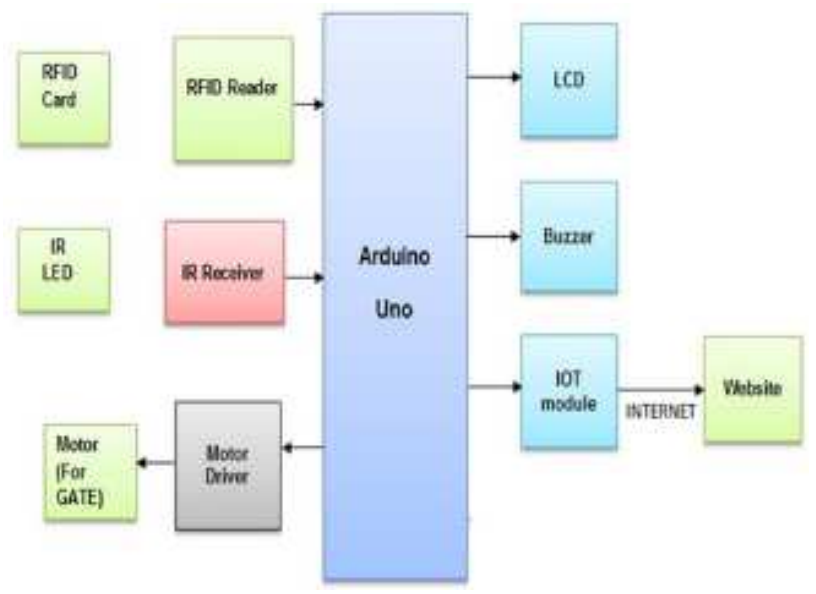

Fig 4: Block diagram of proposed system

\section{EXPERIMENTAL RESULT}

Proteus could be a computer code package for software system, simulation and style of electronic circuits. It consists of 2 main elements, the ISIS, the circuit style setting that conjointly includes the VSM machine and therefore the ARES the PCB designer. Developer and manufacturer is the company Lab center Electronics. Proteus PCB Design Proteus PCB design electronic circuit can computer-aided design and circuit boards are designed. ISIS (Intelligent Input Schematic System) The ISIS Intelligent Input Schematic System (Intelligent Switching input system), is the environment for the design and simulation of Electronic circuits.

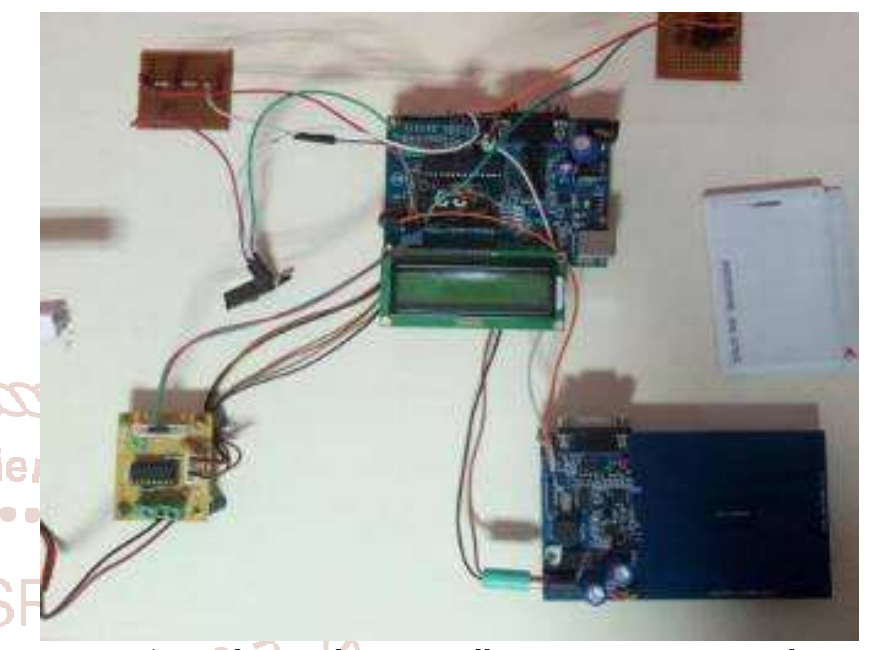

Fig 5: Hardware showing tollgate automation result

Whenever the vehicle enters the tollgate, RFID senses the number of the vehicle, name of the authorized person that will be displayed in the LCD. While entering the tollgate the money will be automatically transacted to the toll booth. Once the amount paid, the gate opens for the concerned vehicle. If the amount is not paid, the gate will not open. Then the data of the vehicles entered into the tollgate will be displayed on the system monitor.

\section{CONCLUSION}

The machine-driven toll assortment system can facilitate in greatly scale back the time needed to complete the method of supplying toll receipts and alter the full system to be swifter. The system additionally eliminates chance of any reasonably human error that may be doable with the traditional toll assortment systems. The system implements Wi-Fi technology within the application of toll legal system. $\mathrm{Wi}-\mathrm{Fi}$ toll assortment stations enable the traffic to flow unceasingly and vehicle having been avoided stopping and beginning once more. This together with reduced fuel consumption has positive impact on atmosphere i.e. pollution created are going to be minimum. Implementing the Wi-Fi technology is additionally not thus expensive. IoT based toll booth monitoring system is an Arduino based toll collection system. The results obtaine $d$ from working have shown that the system performance is quite reliable. The system has successfully overcome the shortcomings of the existing system by reducing the man power at the toll booth. It increases safety in public transport and Change in travel patterns for upcoming generations. In future, this system will be extended tol collect all information of the vehicle, that database will be saved by using cloud computing and the theft vehicle information send to owner of the vehicle and also send to the higher officials. 


\section{REFERENCES}

[1] Neil Kolban,"KOLBAN'S BOOK ON ESP8266”, January 2016.

[2] Olivier Hersent, David Boswarthick, Omar Elloumi, "The Internet of Things - Keyapplications and Protocols", Wiley Publication, Student Edition, Reports:

[3] Kamarulazizi K., Ismail W., "Electronic Toll Collection System Using Passive RFID Technology". Journal of Theoretical and Applied Information Technology.

[4] S. Nandhini, P. Premkumar,"Automatic Toll Gate System Using Advanced RFID and GSM Technology," IEEE Transactions on Signal Processing, Vol.3, Issue 11, November-2014.

[5] K. Senthil, Choudhury R., Basavaraju S., "Automated Toll Collection System using NFC", SSRG International
Journal of Computer Science and Engineering, Vol 4, Issue 3, March-2017.

[6] Atif Ali Khan a, Adnan I. ElberjaouiYakzan b, DrMaaruf Ali c, "Radio Frequency Identification (RFID) Based Toll Collection System", IEEE 2011 Third International Conference on Computational Intelligence, Communication Systems and Networks,2011.

[7] Aniruddha Kumawat, Kshitija Chandramore, "Automatic Toll Collection System Using RFID”,International Journal of Electrical and Electronics Research ISSN 2348-6988 (online)

[8] P Wenter, Siemens AG, Germany, "Automatic Fee Collection Ongerman Autobahns - The Chip ticket System", IEEE. 